\title{
Stiffness optimization of a cable driven parallel robot for additive manufacturing *
}

\author{
D. Gueners, H. Chanal and B.C. Bouzgarrou
}

\begin{abstract}
In this paper, the optimization of the anchor points of a cable driven parallel robot (CDPR) for 3D printing is proposed in order to maximize the rigidity. Indeed, in the context of 3D printing, robot stiffness should guarantee a high level of tool path following accuracy. The optimized platform showed a rigidity improvement in simulation, but also experimentally with a first study of vibration modes. In the same time, this study illustrates the influence of preload in cables on the platform rigidity.
\end{abstract}

\section{INTRODUCTION}

One of the main advantages of CDPRs [1] is their low mass in motion and their large workspace. For 3D printing, the printing speed may be high. The use of a cable robot for additive manufacturing is therefore relevant. However, at present, their accuracy is inferior to that of rigid robots mainly due to the flexibility of the cables. The rigidity also depends on the position of the anchor points on the mobile platform and on the fixed frame. Another problem to consider in designing a CDPR for 3D printing is the collision between the cables and the part to be printed which restricts the printing space.

In [2], 2 algorithms for collision detection between cables of a suspended robot and its environment using interval analysis is proposed. In [3], a trajectory generator is proposed to avoid obstacles for robot suspended on 4 cables. In [4], the printing space of a totally constrained 8-cable is computed in order to take into account the collisions between the cables and the workpiece. The obtained printing space has a volcano shape. In [5], the anchor points of a parallel suspended cable robot have been optimized to increase the workspace for heavy load handling. In [6], the anchor points are optimized to follow the movements of human walking with two platforms attached to the soles of the feet. In [7], a suspended CDPR was used for $3 \mathrm{D}$ printing.

These different articles did not take into account explicitly the rigidity in their optimization. In the case of a cable parallel robot totally constrained for additive manufacturing, the printing space is scientifically smaller than the robot's workspace. This paper proposes to optimize the rigidity in a given size of the robot printing space. Indeed, for 3D printing, it is necessary to perform a high accuracy trajectory tracking. To satisfy a good tracking accuracy in high speed, it is necessary to satisfy a good level of rigidity. The proposed research is carried out within the $\mathrm{CABFAB}$ project, which addresses the design of a CDPR for additive manufacturing processes. This paper is the continuation of the work published

\footnotetext{
* Research supported by the Auvergne-Rhône-Alpes Region as part of the 2017 Ambition Pack Research program
}

in [8] where the stiffness matrix is detailed and a first analysis of the influence of the cable preloads has been carried out.

This paper proposes an algorithm for optimizing the rigidity for a given workspace size of a fully constrained CDPR for 3D printing. Next, the results on rigidity and vibration modes are analyzed between the non-optimized and optimized platform. The robot's dynamic accuracy is evaluated between the different configurations. Finally, the results are validated on an experimental setup.

\section{ANCHORS POINTS OPTIMIZATION FOR EXTRUDING ADDITIVE MANUFACTURING}

Material extrusion-based additive manufacturing mainly consists in deposing layers of fused filament on a horizontal plane. For this technology, the main degrees of freedom are the 3 translations according to $\mathrm{x}, \mathrm{y}$ and $\mathrm{z}$. We have chosen to make the optimization of the anchor points in the case where the deposit of filament must be horizontal. The overall space of the robot is $1 \mathrm{~m}^{3}$. The different points and vectors are represented in figure 1 . The fixed frame of reference is $\mathcal{R}_{O}\left(O, \mathbf{e}_{x_{O}}, \mathbf{e}_{y_{O}}, \mathbf{e}_{z_{O}}\right)$ and the mobile platform frame is $\mathcal{R}_{E}\left(C, \mathbf{e}_{x_{E}}, \mathbf{e}_{y_{E}}, \mathbf{e}_{z_{E}}\right) . A_{i}$ are the anchor points on the mounting frame and $B_{i}$ are the anchor points on the mobile platform.

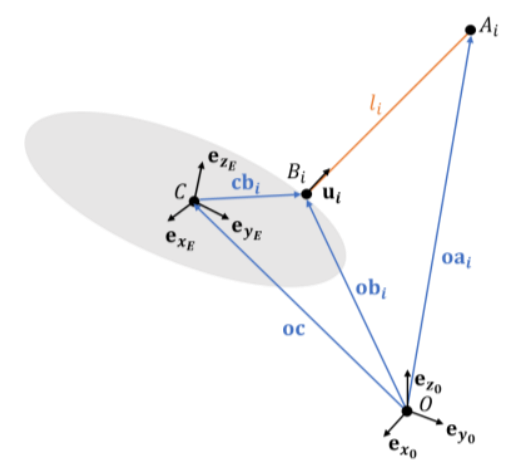

Figure 1. Schematic representation of one cable

The robot workspace should be computed thanks to a robot geometric behavior model and a printing part collision detection algorithm.

\section{A. Calculation of the intersection point between a cable and a horizontal plane}

This work allows to determine the workspace where cables have no collision with the printed part. To do so, we compute the size of cylinders without cable collision.

All the authors is with University Pascal, CNRS, Sigma Clermont, Institut Pascal in Clermont-Ferrand, FRANCE (D. Gueners can be reach as corresponding author at damien.gueners@ sigma-clermont.fr). 
The cables being thin and having negligible mass, they can be likened to segments with anchors points $A_{i}$ and $B_{i}$ as ends. The set of points on a cable segment must satisfy the equation:

$$
\left\{\begin{array}{l}
x=a t+x_{a} \\
y=b t+y_{a}, \text { where } t \in[0 ; 1] . \\
z=c t+z_{a}
\end{array}\right.
$$

with $\left(x_{a}, y_{a}, z_{a}\right)$ are the coordinates anchor point $A_{i}$, $(a, b, c)$ are the components of the director vector $\mathbf{a}_{i} \mathbf{b}_{i}^{0}$, $(x, y, z)$ are the coordinates of a point of the segment, all expressed in the origin frame $\mathcal{R}_{O}$. Let $C_{i}$ be the intersection between the segment $\left[A_{i} B_{i}\right]$ and the horizontal plane situated at a height $h$. Its coordinates can be calculated by the equation:

$$
\left\{\begin{array}{c}
x=a \frac{h-z_{a}}{c}+x_{a} \\
y=b \frac{h-z_{a}}{c}+y_{a} \\
z=h
\end{array} .\right.
$$

\section{B. Detection of collisions between cables and a cylindrical surface}

The printing part is supposed to be included in a cylinder of radius $R$ and delimited by two horizontal discs at heights $z=0$ and $z=h$. The collision detection algorithm between the cables and the upper circular surface of the cylinder is proposed below. It returns TRUE if there is an intersection between the cable segments and the circular surface. First, the intersection point $C_{i}$, between the cable and the horizontal plane at a height $h$ is calculated. If this point is on the segment $\left[A_{i} B_{i}\right]$ and its position in the plane is within the upper cylinder disc of radius $R$, then the cable collides with the part. If no collision has been detected, it returns FALSE. In our application, the position of the printing nozzle is always above the horizontal plane located at height $h$ and inside the cylinder of radius $R$. This means that if there is contact with the sidewall of the cylinder, there is also contact with the upper face of the cylinder.

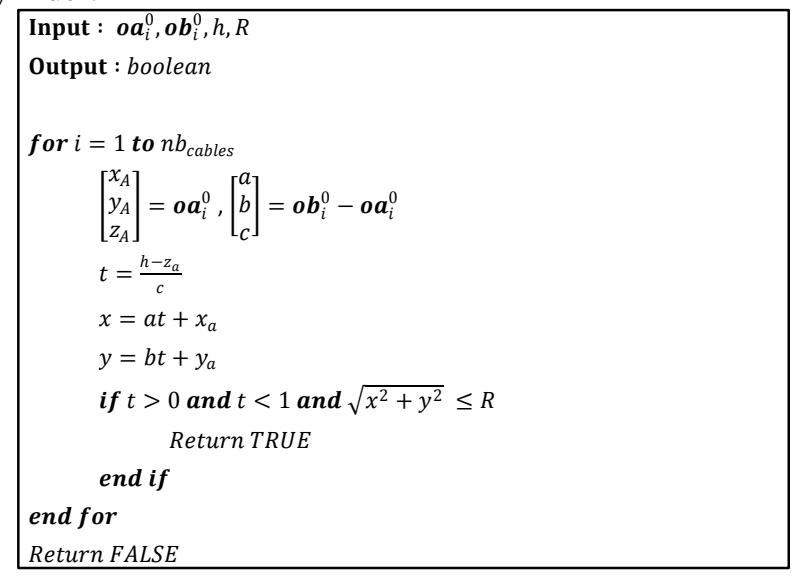

Algorithm 1. Detection of collisions between cables and a cylindrical surface

\section{Maximum printing space}

In our case, the possible printing space is dependent on the collisions between the cables and the printed part. The algorithm 2 calculates the possible printing space.

It takes as input the reachable working space of the robot $\mathcal{L}_{\text {workspace }}$, minimum and maximum radius $R_{\text {min }}$, and $R_{\text {max }}$ of the acceptable cylinder (without collision) as well as its minimum and minimum heights $h_{\min }$ and $h_{\max }$, and the positions of the anchors points $\boldsymbol{o a _ { i } ^ { 0 } , \boldsymbol { o b }} \boldsymbol{b}_{i}^{0} \cdot R_{\text {step }}$ and $h_{\text {step }}$ are respectively the discretization steps for the radius and the height, chosen equal in this case. Each iteration calculates the maximum height for a printable cylinder of radius $R$. The algorithm first creates a cylinder of radius $R$ in the $\mathcal{L}_{\text {workspace }}$. The points included in the cylinder are stored in the list $\mathcal{L}_{\text {cylinder. }}$ It then computes for all the positions included in the cylinder if there is contact between the cables and the upper surface of the cylinder. The printing is layer by layer, so we must guarantee that there is no collision with the lower printed layers, that is to say from $h_{\min }$ to $h_{\max }$. The maximum height is calculated using the end position of the print nozzle $\boldsymbol{o p} \boldsymbol{p}^{0}$. If there is a collision, the position index is saved. Positions for which there has been no collision $\left(\mathcal{L}_{\text {cylinder }} \backslash\right.$ $\mathcal{L}_{\text {cylinder }}($ indice)) are stored in the printing space list $\left(\mathcal{L}_{\text {printable }}\right)$. The total print volume will represent the union of cylindrical volumes as shown in the figure 2 . The process repeatedly calculates the center region where all the cylinders overlap. This could increase the computation time but the algorithm complexity is reduced. Computation time was not a criterion in the writing of the algorithm.

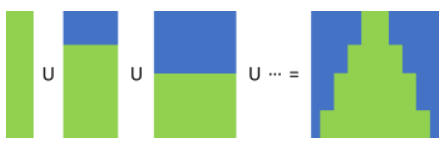

Figure 2. Printing space.

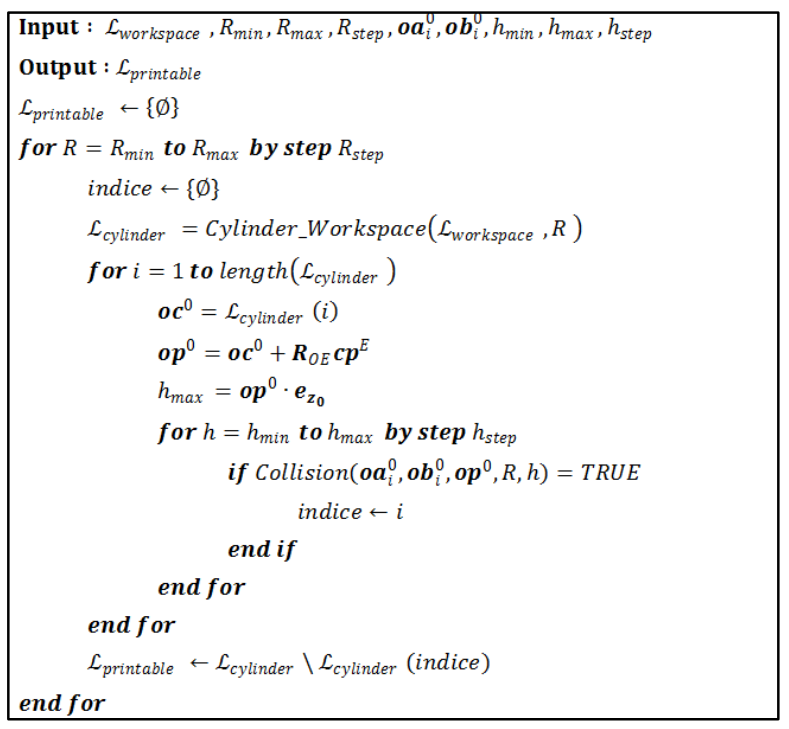

Algorithm 2. Printing space construction

\section{Optimization parameters}

The optimized robot has 6 degrees of freedom and 8 cables. The anchor points on the effector (figure 3) are distributed on two circles located on 2 parallel planes at a distance $h$ from each other. 6 design parameters are proposed to optimize the anchor points on the mobile platform:

- $\quad h_{p}$ : the height between the upper and the lower planes;

- $r_{h}$ : the radius of the upper face;

- $r_{b}$ : the radius of the lower face;

- $\theta$ : the angle between the reference of the upper and lower face; 
- $\theta_{h}$ : the angle between the anchor points of the upper face;

- $\theta_{b}$ : the angle between the anchor points on the underside.

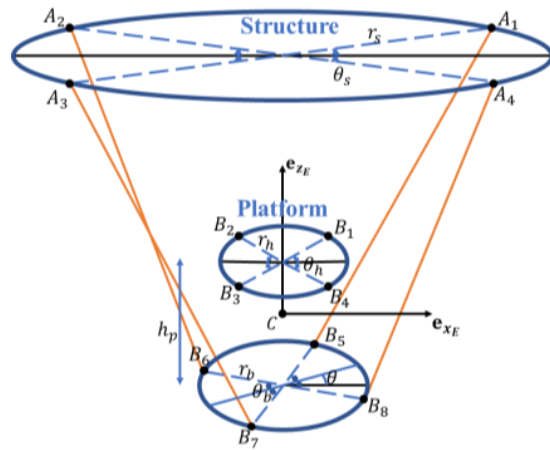

Figure 3. Optimization settings.

The cables coming from the bottom of the structure (fixed base) are connected to the anchor points of the top of the effector (mobile platform). The cables coming from the top of the structure are connected to the anchor point of the bottom of the effector.

The fixed frame is defined as a polar coordinate system. The anchor points of the lower surface are fixed in square. The upper surface is defined by 2 parameters:

- $r_{S}$ : the radius of the upper face structure;

- $\theta_{s}:$ the angle between the anchor points on the upper face of the fixed base.

We therefore have 8 design parameters.

\section{E. Optimization algorithm}

Two-stage optimization is achieved:

- $\quad$ a cost function on the printing space with a constraint function on the first mode of vibration;

$$
\left\{\begin{array}{c}
f_{\text {cost }}=V_{\text {workspace }} / V_{\text {printing_space }} \\
f_{\text {constraint }}>f 1_{\text {min }}
\end{array}\right.
$$

- a cost function on the first modal frequency, to be maximized, with a constraint function on the printing space.

$$
\left\{\begin{array}{c}
f_{\text {cost }}=f 1_{\text {min }} \\
f_{\text {constraint }}>V_{\text {print_min }}
\end{array}\right.
$$

The first step increases the printing space. The cost function tries to make the printing space closest to the workspace, i.e. minimizing the volume ratio of the workspace on the printing space. To ensure a good trajectory follow, it is necessary to ensure a sufficient level of rigidity of the structure The rigidity of the structure will be greater if the 1st mode is higher. The constraint function imposes a minimum frequency greater than $10 \mathrm{~Hz}$.

The second step increases the rigidity in the printing space while maintaining its volume close to that computed in the first step. In fact, the results of the first step are used as initial configuration of the second step. The cost function is the inverse of the frequency of the first mode. The constraint function imposes as minimum printing space that calculated during the first step.

\section{RESULTS}

The optimization started with rectangular parallelepiped shaped mobile platform according to CDPR studied in the literature. The rigidity associated with this configuration has been analyzed in [8]. The fixed base is a cube shaped of $1 \mathrm{~m}^{3}$. The second step use optimal design found by the first step. The 'fmincon' function of Matlab was used to optimize the cost functions with 2 algorithms 'active set' and 'sqp'. A step of $0.02 \mathrm{~m}$ was used and the limits of a cube of $1 \mathrm{~m} 3$. The position of the end of the nozzle on the effector is $\boldsymbol{o p} \boldsymbol{p}^{0}=\left(\begin{array}{lll}0 & 0-0.1\end{array}\right)^{t}$. The calculation time was near 24 hours. Table 1 shows the optimization results on the design parameters.

The effector optimized after the second step is a plate with the anchor points on the outer edges. They form rectangles of different sizes. Anchor points for the cables coming from the bottom form a rectangle oriented relatively to the reference frame by an angle of $29.98 \mathrm{deg}$ and lie on a circle of a radius of $0.1 \mathrm{~m}$. Anchor points for the cables coming from the top form a rectangle oriented by an angle of $15.87 \mathrm{deg}$ and lie on a circle of a radius of $0.1 \mathrm{~m}$. The positions of the anchor points on the structure are little modified with a shift of $13.3 \mathrm{~mm}$ between the optimized configuration and the initial one. The initial printing space represents $5.5 \%$ of the overall robot space $\left(1 \mathrm{~m}^{3}\right)$, while for the optimization, it almost doubled with $9.6 \%$ after the second step. The printing space is mainly constrained by the cables at the bottom of the robot (which potentially collide with the part to be printed).

TABLE I. PARAMETER

\begin{tabular}{|l|l|l|l|}
\hline Parameter & Initial & Step 1 & Step 2 \\
\hline$h_{p}(\mathrm{~m})$ & 0.05 & 0 & 0 \\
\hline$r_{h}(\mathrm{~m})$ & 0.0623 & 0.1 & 0.1 \\
\hline$r_{b}(\mathrm{~m})$ & 0.0623 & 0.045 & 0.1 \\
\hline$\theta(\mathrm{deg})$ & 0 & 0 & 0 \\
\hline$\theta_{h}(\mathrm{deg})$ & 40.2 & 31.7 & 29.98 \\
\hline$\theta_{b}(\mathrm{deg})$ & 40.2 & 20.2 & 15.87 \\
\hline$r_{s}(\mathrm{~m})$ & 0.7071 & 0.6924 & 0.6938 \\
\hline$\theta_{s}(\mathrm{deg})$ & 45 & 45 & 45 \\
\hline
\end{tabular}

The following figures show the rigidities (figure 4) and modal frequencies (figure 5) of the different configurations for an equivalent $69.5 \mathrm{GPa}$ Young's modulus steel cable and a diameter of $0.54 \mathrm{~mm}$ without a preload. The setting of the rotations is as follows: $\psi$ is a rotation about $\mathbf{e}_{x_{0}}, \theta$ is a rotation about $\mathbf{e}_{y_{0}}$ and $\varphi$ is a rotation about $\mathbf{e}_{z_{0}}$.

\section{A. Stiffness analysis}
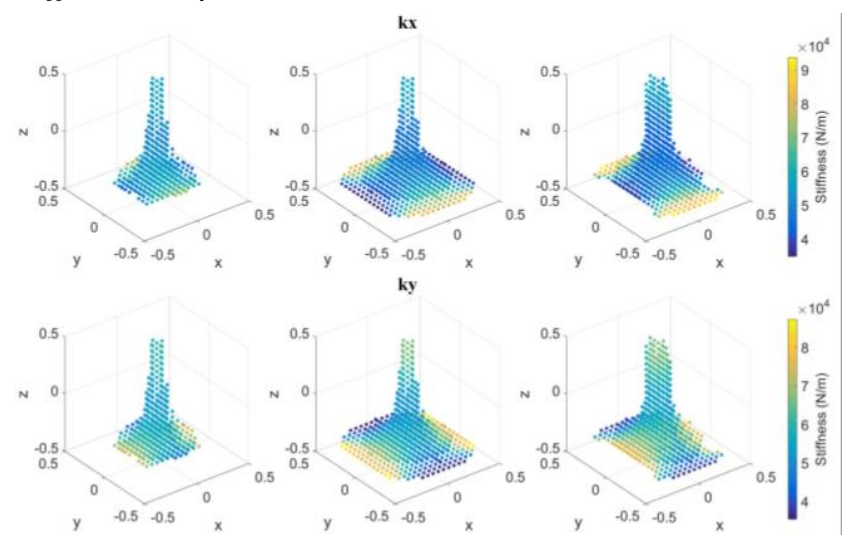

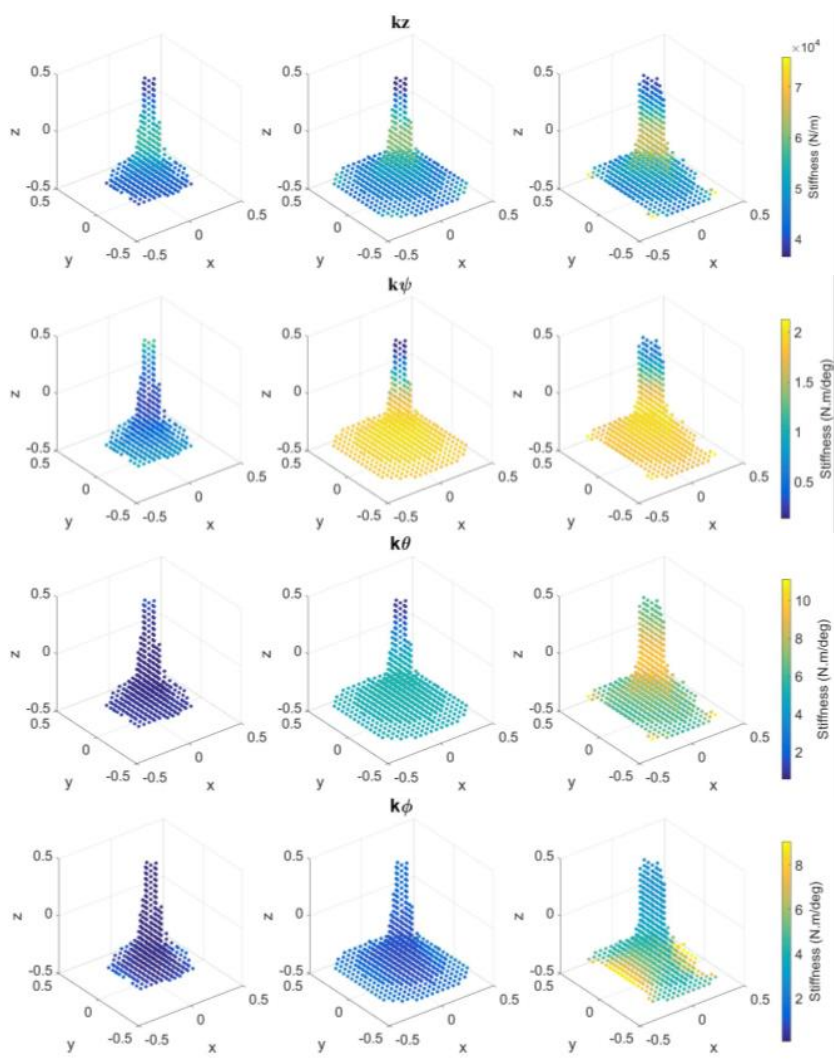

Figure 4. Printing space stiffness on the $6 \mathrm{DoF}$ for the initial (left), optim1 (center) and optim2 (right) configurations.

TABLE II. STIFFNESS RESULTS

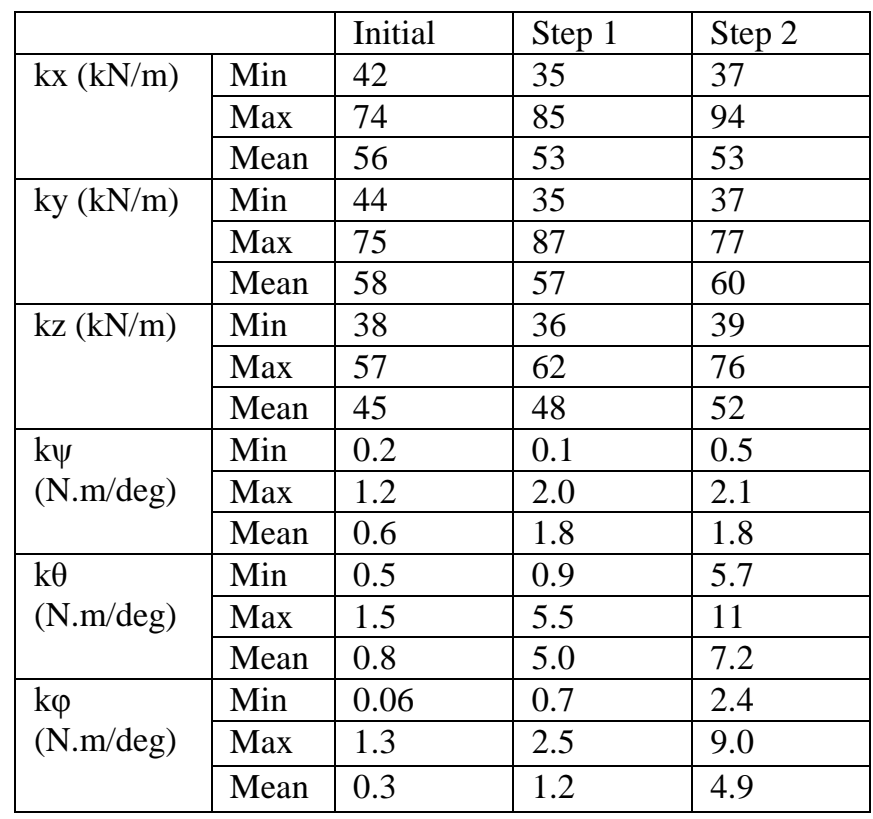

The stiffness according to $\mathrm{x}, \mathrm{y}$ and $\mathrm{z}$ are globally similar, with an average of about $53-56 \mathrm{kN} / \mathrm{m}$ for $\mathrm{kx}, 58-60 \mathrm{kN} / \mathrm{m}$ for ky and $45-52 \mathrm{kN} / \mathrm{m}$ for kz for the different configurations. The main changes concern orientation stiffness. The average stiffness according to $\psi$ increased from $0.6 \mathrm{~N} . \mathrm{m} / \mathrm{deg}$ to 1.8 N.m/deg between the initial design and the optimized design. The average of the stiffness according to $\theta$ has passed from 0.8 N.m/deg to $7.2 \mathrm{~N} . \mathrm{m} / \mathrm{deg}$ between the initial and the optimized design. The average stiffness according to $\varphi$ has increased from $0.3 \mathrm{~N} . \mathrm{m} / \mathrm{deg}$ to $4.9 \mathrm{~N} . \mathrm{m} / \mathrm{deg}$ between the initial design and the second optimized design. Table 2 summarizes stiffness optimization results.

\section{B. Modal analysis}

For vibration modes, we note that the 6 modal frequencies have been increased. The first modal frequency increased from $5.86 \mathrm{~Hz}$ to $14.29 \mathrm{~Hz}$ with the first optimization and to $19.31 \mathrm{~Hz}$ with the second optimization. The average of the first modal frequency overall the printing space is $30.05 \mathrm{~Hz}$ after optimization. The first mode is the most important in the center of the printing area as well as the second mode which are similar. The distribution of the modes is different between the initial and the optimal design. The mobile platform has been more rigidified in the central printing area. Table 3 summarizes of the optimization results on modal frequencies.
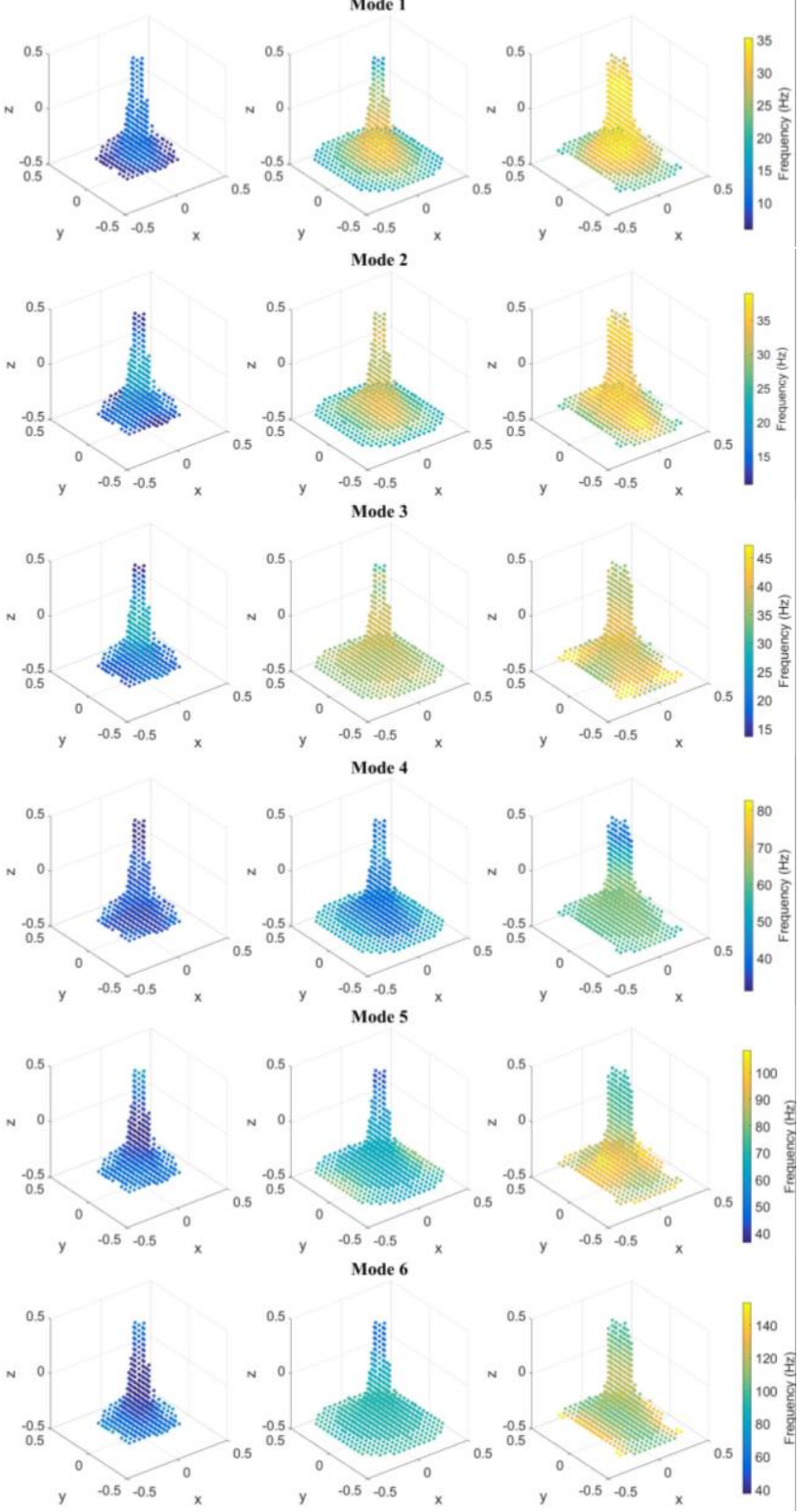

Figure 5. Printing space modal for the initial (left), optim1 (center) and optim2 (right) configurations. 
TABLE III. MODAL RESULT

\begin{tabular}{|c|c|c|c|c|}
\hline & & Initial & Step 1 & Step 2 \\
\hline \multirow{3}{*}{$\begin{array}{l}\text { Mode } 1 \\
(\mathrm{~Hz})\end{array}$} & Min & 5.86 & 14.29 & 19.31 \\
\hline & Max & 11.44 & 33.97 & 35.35 \\
\hline & Mean & 9.20 & 25.33 & 30.05 \\
\hline \multirow{3}{*}{$\begin{array}{l}\text { Mode } 2 \\
(\mathrm{~Hz})\end{array}$} & Min & 10.76 & 21.96 & 24.90 \\
\hline & Max & 22.16 & 35.01 & 38.97 \\
\hline & Mean & 15.95 & 29.22 & 33.43 \\
\hline \multirow{3}{*}{$\begin{array}{l}\text { Mode } 3 \\
(\mathrm{~Hz})\end{array}$} & Min & 13.58 & 30.40 & 34.33 \\
\hline & Max & 29.06 & 39.32 & 47.26 \\
\hline & Mean & 20.78 & 36.60 & 38.99 \\
\hline \multirow{3}{*}{$\begin{array}{l}\text { Mode } 4 \\
(\mathrm{~Hz})\end{array}$} & Min & 31.03 & 36.89 & 39.63 \\
\hline & Max & 40.78 & 54.74 & 65.52 \\
\hline & Mean & 35.47 & 44.16 & 59.29 \\
\hline \multirow{3}{*}{$\begin{array}{l}\text { Mode } 5 \\
(\mathrm{~Hz})\end{array}$} & Min & 36.40 & 40.35 & 68.07 \\
\hline & Max & 59.77 & 82.82 & 108.35 \\
\hline & Mean & 46.80 & 66.24 & 88.49 \\
\hline \multirow{3}{*}{$\begin{array}{l}\text { Mode } 6 \\
(\mathrm{~Hz})\end{array}$} & Min & 37.48 & 51.96 & 98.35 \\
\hline & Max & 73.58 & 101.13 & 153.74 \\
\hline & Mean & 51.95 & 89.80 & 113.94 \\
\hline
\end{tabular}

\section{Dynamic simulation}

The initial and optimal configuration are compared in dynamic simulation for a circular trajectory of radius $0.3 \mathrm{~m}$ at a height $\mathrm{z}=-0.2 \mathrm{~m}$ with a duration of 2 seconds. The dynamic model is the same as in [8]. A force distribution algorithm in the cables was used with a minimum preload of $1 \mathrm{~N}$ and a maximum of $100 \mathrm{~N}$. Details on the force distribution algorithm can be found in [9]. Circular (figure 6), z (figure 7) and orientation errors (figure 8) are shown in the figures below.

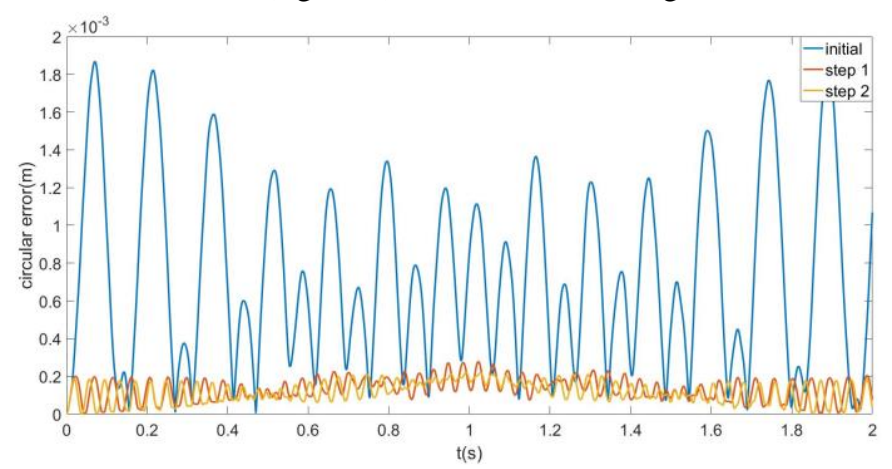

Figure 6. Circular error function of te time.

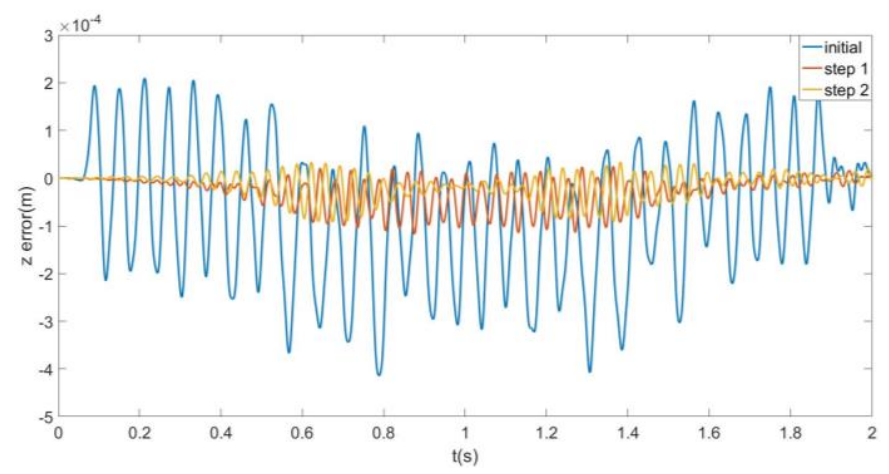

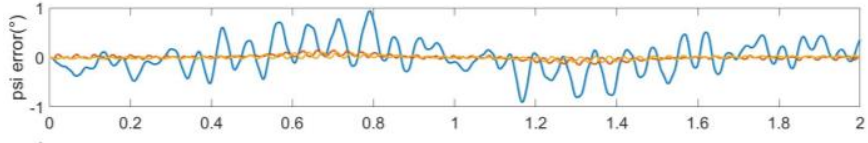

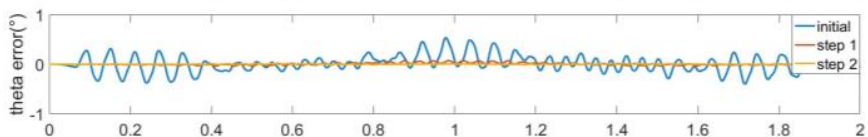

$\stackrel{\Phi}{E}$

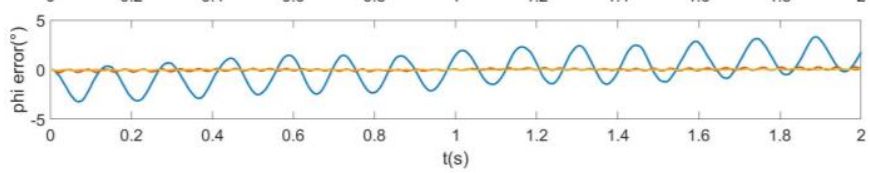

Figure 8. Orientation error function of the time.

The errors of the optimal design are lower than the initial configuration. The second optimization step has better performance than the first optimization step for the simulated trajectory. The maximum circular error is $1.869 \mathrm{~mm}$ for the initial configuration and $222 \mu \mathrm{m}$ for the second optimal configuration. The altitude error goes from $414 \mu \mathrm{m}$ to $92 \mu \mathrm{m}$. The error in orientation has been greatly decreased. The maximum orientation error according to $\psi$ is $0.94 \mathrm{deg}$ for the initial configuration, $0.16 \mathrm{deg}$ for the first optimization step and $0.11 \mathrm{deg}$ for the second optimization step. The maximum error according to $\theta$ is 0.54 deg for the initial configuration, $0.08 \mathrm{deg}$ for the first optimization step and $0.02 \mathrm{deg}$ for the second optimization step. The maximum error according to $\varphi$ is $3.31 \mathrm{deg}$ for the initial configuration, $0.28 \mathrm{deg}$ for the first optimization step and 0.10 deg for the second optimization step. Table 4 is a summary of maximal error.

TABLE IV. MAXIMAL ERROR

\begin{tabular}{|l|l|l|l|}
\hline & Initial & Step 1 & Step 2 \\
\hline $\mathrm{X}(\mu \mathrm{m})$ & 1183 & 279 & 210 \\
\hline $\mathrm{Y}(\mu \mathrm{m})$ & 1868 & 233 & 210 \\
\hline $\mathrm{Z}(\mu \mathrm{m})$ & 414 & 117 & 92 \\
\hline Psi $(\mathrm{deg})$ & 0.94 & 0.16 & 0.11 \\
\hline Theta $(\mathrm{deg})$ & 0.54 & 0.08 & 0.02 \\
\hline Phi $(\mathrm{deg})$ & 3.31 & 0.28 & 0.10 \\
\hline
\end{tabular}

The optimization of the anchors points contributes greatly to improve the accuracy of the CDPR in toolpath following. The volume of the printing space has been slightly increased. The choice of a fully constrained parallel cable robot solution greatly improves rigidity at the border of printing space.

\section{EXPERIMENTAL VALIDATION}

To validate rigidity optimization experimentally two platforms are studied:

- The first corresponds to the initial design of the mobile platform used in the optimization algorithm;

- The second is the optimal platform after the second optimization step.

The anchor points on the fixed structure having little difference regarding the simulated configurations. They are the same for both platforms. The experimental device is a Leica laser traker which ensure to measure the position and 
orientation of the platform with an accuracy of $21 \mu \mathrm{m}$ at $1 \mathrm{~m}$ of the measurement. Force sensors are attached at the end of each cable. The vibratory study is carried out by using impact testing device and the software 'Siemens Simcenter Testlab' for modal extraction. Two triaxial accelerometers are used to obtain the movements of the platform. An impact hammer is used to apply an impulse excitation. The cables are $0.54 \mathrm{~mm}$ diameter braided steel from Carl Stahl. Photo of the experimental setup can be seen on the figure 9 .

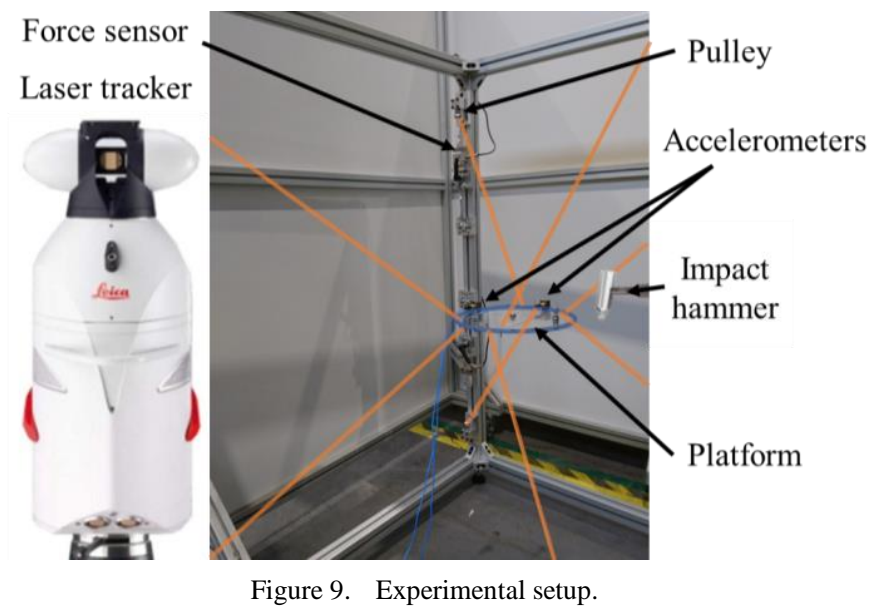

The influence of the preloads in the cables is studied for the 2 platforms (figure 10) with a $2.4 \mathrm{~kg}$ cylindrical mass. The platform is close to the CDPR center with zero orientation. The evolution of the 6 modal frequencies according to the average preload in the cables can be seen in the figures 11 for the initial design and figure 12 for the optimal design.
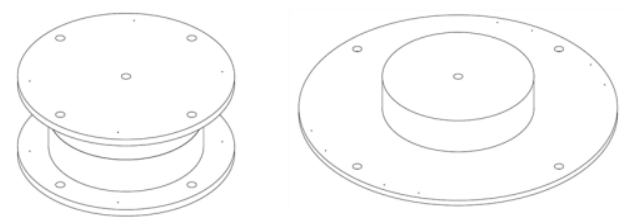

Figure 10. Sketches of the initial design (left) and the optimal design (right)

For the initial platform, the first mode is at a frequency of $8.4 \mathrm{~Hz}$ for an average preload of $15 \mathrm{~N}$ in the cables. It increases $111 \%$ with an average preload of $85 \mathrm{~N}$, a frequency of 17.6 $\mathrm{Hz}$. The first mode corresponds to a deformed in rotation around the vertical axis. For the other modes, the preload influence is less important than the first mode. The frequencies have increased by $4.5 \%$ overall between an average preload of $15 \mathrm{~N}$ and $85 \mathrm{~N}$.

For the optimal platform, the frequency of the first mode is at $20 \mathrm{~Hz}$ for an average preload of $12.5 \mathrm{~N}$ in the cables. The frequency of the first mode of the optimal platform is clearly higher than that of the initial platform for equivalent force in the cables. As expected, the optimized platform has a better rigidity than the initial platform. However, the influence of the preload in the cables is less important with the optimized platform. But, there is an increase of the 5th mode of $14.2 \%$. The frequency of the 5 th mode is $37.3 \mathrm{~Hz}$ for an average force of $12.5 \mathrm{~N}$ and $42.6 \mathrm{~Hz}$ for an average force of $83 \mathrm{~N}$. The 5 th mode shape corresponds to a rotation around the vertical axis.
The average preload in the cables seem to increase mainly the rotational stiffness around the vertical $\mathrm{Z}$ axis.

The rigidity of the initial platform clearly depends on the cables preloads. This type of platform requires more complex strategy to control the force in the cables. It must also use more powerful motors capable of supplying significant torque to generate high preload.

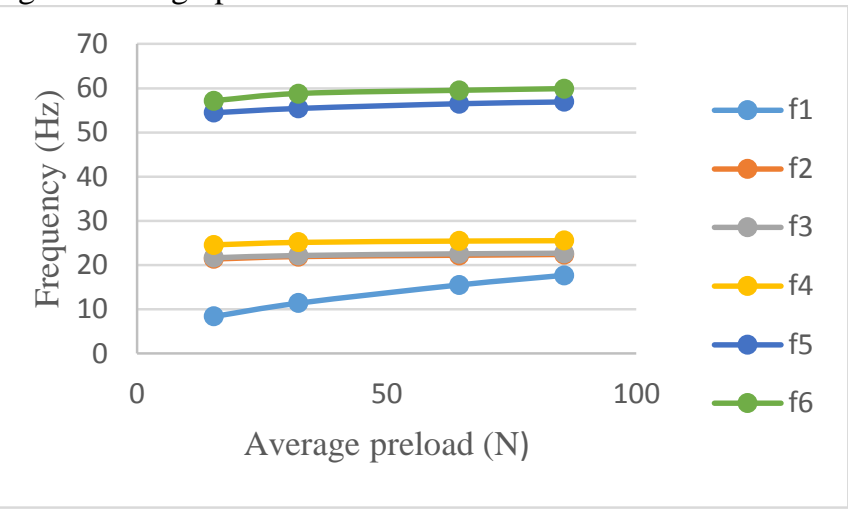

Figure 11. Modal frequencies in function of the cable preload average for the initial design.

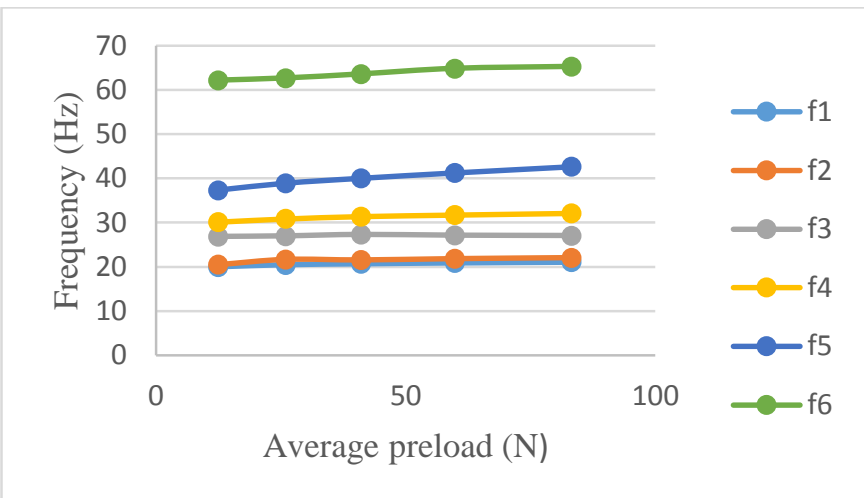

Figure 12. Modal frequencies function of the cable preload average for the optimal design.

\section{CONCLUSION}

An algorithm for optimizing the rigidity and the printing space of a fully constrained CDPR has been proposed. It uses the coordinates of the anchor points on the mobile platform as design variables. Optimized anchor points show better rigidity. Optimization and simulation results have been validated experimentally. Moreover, we have shown that the cable preloads have a significant influence on the rigidity for the two design solutions. In particular, on the rotational rigidity along the vertical axis.

An elastic cable model considering preload effect is under development based on the presented static and dynamic characterizations. Future work will focus on the exploitation of the current results for CDPR controller design for $3 \mathrm{D}$ printing applications.

\section{ACKNOWLEDGMENT}

This paper is part of the CABFAB project funded by the Auvergne-Rhône-Alpes region as part of the 2017 Research Ambition Pack program. 


\section{REFERENCES}

[1] C. Gosselin, "Cable-driven parallel mechanisms: state of the art and perspectives," Mech. Eng. Rev., vol. 1, no. 1, pp. DSM0004-

DSM0004, 2014.

[2] L. Blanchet and J. P. Merlet, "Interference detection for cable-driven parallel robots (CDPRs)," IEEE/ASME Int. Conf. Adv. Intell. Mechatronics, AIM, pp. 1413-1418, 2014.

[3] S. Lahouar, E. Ottaviano, S. Zeghoul, L. Romdhane, and M. Ceccarelli, "Collision free path-planning for cable-driven parallel robots," Rob. Auton. Syst., vol. 57, no. 11, pp. 1083-1093, 2009.

[4] M. Fabritius and C. Martin, "Calculation of the Collision-Free Printing Workspace for Fully-Constrained Cable-Driven Parallel Robots," 2018, pp. $1-9$.

[5] M. Gouttefarde, J. F. Collard, N. Riehl, and C. Baradat, "Geometry Selection of a Redundantly Actuated Cable-Suspended Parallel Robot," IEEE Trans. Robot., vol. 31, no. 2, pp. 501-510, 2015.

[6] S. Perreault and C. M. Gosselin, "Cable-Driven Parallel Mechanisms: Application to a Locomotion Interface," J. Mech. Des., vol. 130, no. 10, p. 102301, 2008.

[7] E. Barnett and C. Gosselin, "Large-scale 3D printing with a cablesuspended robot," Addit. Manuf., vol. 7, pp. 27-44, 2015.

[8] D. Gueners, B. Chedli Bouzgarrou, and H. Chanal, "Static and dynamic analysis of a 6 DoF totally constrained cable robot with 8 preloaded cables," in Mechanisms and Machine Science, 2019, vol. 74, pp. 307318.

[9] A. Pott, T. Bruckmann, and L. Mikelsons, "Closed-form Force Distribution for Parallel Wire Robots," in Computational Kinematics, 2009, pp. 25-34. 